Research Paper

\title{
High expression of F2RL3 correlates with aggressive features and poor survival in clear cell renal cell carcinoma
}

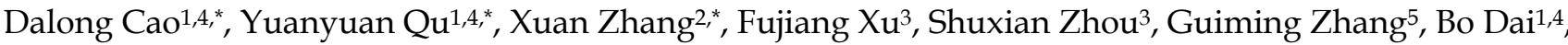

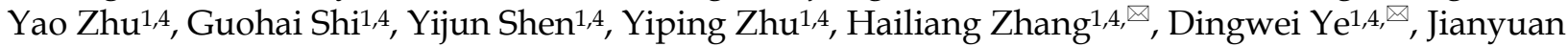 \\ Zhao ${ }^{2, \mathbb{}}$ \\ 1. Department of Urology, Fudan University Shanghai Cancer Center, Shanghai 200032, China \\ 2. The State Key Laboratory of Genetic Engineering and Collaborative Innovation Center of Genetics \& Development, School of Life Sciences, Fudan \\ University, Shanghai 200433, China \\ 3. Institutes of Biomedical Sciences, Fudan University, Shanghai 200032, China \\ 4. Department of Oncology, Shanghai Medical College, Fudan University, Shanghai 200032, China \\ 5. Department of Urology, The affiliated hospital of Qingdao University, Shandong, 266071, China \\ "These authors contributed equally to this work.
}

$\triangle$ Corresponding authors: Dingwei Ye, M.D.; Department of Urology, Fudan University Shanghai Cancer Center, No.270 Dong'an Road, Shanghai 200032, China; Tel: 86-21-64175590-81807; Fax: 86-21-64434556; E-mail: dwyeli@163.com; Jianyuan Zhao, Ph.D.; The State Key Laboratory of Genetic Engineering and Collaborative Innovation Center of Genetics \& Development, School of Life Sciences, Fudan University, No.2005 Songhu Road, Shanghai 200433, China; Tel: 86-21-51630421; E-mail: zhaojy@fudan.edu.cn; Hailiang Zhang, M.D.; Department of Urology, Fudan University Shanghai Cancer Center, No.270 Dong'an Road, Shanghai 200032, China; Tel: 86-21-64175590-82800; Fax: 86-21-64434556; E-mail: zhangh1918@163.com

(C) Ivyspring International Publisher. This is an open access article distributed under the terms of the Creative Commons Attribution (CC BY-NC) license (https://creativecommons.org/licenses/by-nc/4.0/). See http://ivyspring.com/terms for full terms and conditions.

Received: 2017.11.19; Accepted: 2018.07.25; Published: 2018.09.08

\begin{abstract}
Background: Specific lifestyle factors including tobacco-exposure are vital etiologic factors for renal cell carcinoma (RCC), F2R Like Thrombin/Trypsin Receptor 3 (F2RL3) is associated with smoking but it is unknown whether its expression translate into poor survival of clear cell RCC (ccRCC). In the current study, the expression profiling and prognostic value of F2RL3 in Chinese patients with ccRCC were investigated.

Methods: Using Quantitative PCR analysis and immunohistochemistry, the relative expression levels of F2RL3 in 367 paired ccRCC and adjacent normal tissues were calculated. Cox regression analysis was used to identify independent prognostic factors and Kaplan-Meier analysis and a log-rank test were employed to evaluate the prognostic value of F2RL3.

Results: We observed that high expression of F2RL3 mRNA and protein were strongly correlated with shorten progression-free survival (PFS) of ccRCC with hazard ratios (HR; $95 \%$ confidence interval $(\mathrm{Cl}))$ of $2.060(1.410-3.009)$ and $1.657(1.193-2.300)$, respectively, as well as with poor overall survival (OS) with $\mathrm{HRs}(95 \% \mathrm{Cl})$ of $2.826(1.713-4.662)$ and $1.712(1.140-2.569)$, respectively. After adjustment for confounding factors including smoking status, elevated $\mathrm{HRs}(95 \% \mathrm{Cl})$ of 2.113 (1.445-3.089) and 1.692 (1.218-2.352) were presented for PFS, respectively, and 2.936 (1.777-4.851) and $1.811(1.203-2.725)$ were present for OS, respectively. Meanwhile, increased F2RL3 mRNA and protein level were reported to significantly associate with smoking-exposure and well-known prognostic factors (higher TNM stage and ISUP grade).

Conclusion: These findings suggested that F2RL3 mRNA and protein level in ccRCC is a robust predictor of poorly prognostic phenotype. Exploring the causal relevance of F2RL3 in ccRCC development further warrants in the future study.
\end{abstract}

Key words: F2RL3 expression, aggressive features, poor survival, renal cell carcinoma 


\section{Introduction}

Kidney cancer is one of the most commonly genitourinary carcinomas, and about $90 \%$ of all kidney cancers are renal cell carcinomas. Approximately 337,860 new cases of kidney cancer were diagnosed, and 143,406 deaths were occurred worldwide in $2012^{[1]}$. It is also statistically estimated that the incidence of kidney cancer in developed regions is higher than that of developing areas. This geographical variation is likely attributed to cancer-related risk factors such as obesity, smoking and hypertension [2]. Especially, tobacco exposure is an established risk factor for kidney cancer. A meta-analysis [3] including 24 studies confirmed that the relative risk (RR) for kidney cancer for frequent smokers compared to never smokers is 1.38. In addition, strong dose-dependent risk increases with heavier smoking and decreases with smokingcessation $>10$ years ${ }^{[3]}$. There also exists evidence that higher exposure to environmental tobacco smoke among never-smokers increases higher risk for kidney cancer [4, 5]. Furthermore, tobacco exposure is associated with poor pathological and clinical stage, and unfavorable prognosis [6, 7]. The established mechanisms that explain how cigarette-smoking leads to carcinogenesis include as follows: (1) exposure to smoking-related carcinogens, (2) formation of DNA adducts between carcinogens and DNA, and (3) mutations induced in critical oncogenes or tumor suppressor genes [8]. For example, 4-(methylnitrosamino)-1-(3-pyridyl)-1-butanone is a carcinogenic chemical present in tobacco. It induces DNA damages-methylation and pyridyloxobutylation adducts that are related with higher risk of kidney cancer [9]. The insights on cancer-related risk factors provide promising avenues for preventing and treating kidney carcinoma.

Coagulation factor II receptor-like 3 gene (F2RL3), coding for protease-activated receptor-4 (PAR4), has been proved to affect platelet activation and cardiovascular functions such as intimal hyperplasia and inflammation ${ }^{[10]}$, and has been investigated as a drug target of cardiovascular importance features ${ }^{[10,11]}$. A single locus in F2RL3 out of 27578 loci tested in the $27 \mathrm{~K}$ discovery and replication study was less methylated in smokers ${ }^{[12]}$. Importantly, methylation levels in F2RL3 were consistently confirmed in several independent studies ${ }^{[12-14]}$. And dose-response associations with F2RL3 methylation were demonstrated for both current and long-term tobacco exposure ${ }^{[15]}$. Furthermore, lower F2RL3 methylation was a stronger predictor of all-cause, cardiovascular, cancer and other mortality ${ }^{[16]}$. In this study, the association of F2RL3 with prognosis of clear cell renal cell carcinoma
(ccRCC), which is the most commonly pathological type of kidney cancer, were evaluated to further understand F2RL3 expression in related to smoking-related disease.

\section{Materials and Methods}

\section{Patients and samples collection}

Three hundred and sixty-seven patients with ccRCC, who underwent radical nephrectomy at Fudan University Shanghai Cancer Center (FUSCC) from 2008 to 2015, were enrolled into this study. Clinical and pathological information (e.g. age at surgery, gender, smoking status, clinical manifestation, laterality, tumor size, ISUP grade and TNM stage) for all participants included in this study were collected and then evaluated (Table 1). Tissue samples (ccRCC and adjacent normal tissues) collected at the time of surgery were obtained from FUSCC tissue bank. Paraffin-embedded specimens preserved at the department of pathology of FUSCC were also obtained for this study. The institutional review board of FUSCC approved this study. Written informed consent for each participant was also obtained.

\section{Quantitative PCR analysis}

Total RNA from 367 paired ccRCC and adjacent normal tissues stored in RNAlater ${ }^{\mathrm{TM}}$ Stabilization Solution (Invitrogen, Carlsbad, CA) were isolated using the TRIzol reagent (Invitrogen, Carlsbad, CA). Upon completion of total RNA collection, reverse-transcribed reaction was performed using SuperScript First Strand cDNA system (Invitrogen, Carlsbad, CA). Real-time PCR was performed using ABI Prism 7900 sequence detection system (Applied Biosystems) with $\beta$-actin as an internal reference. The forward and reverse primers for F2RL3 were $5^{\prime}$-TTC CCTGGAGACTCACTGCAA-3' and 5'-GGTTGCTCA AGAGACATCCCA-3'. For $\beta$-actin gene, the sense and antisense primers were $5^{\prime}$-GCCGACAGGATGCA GAAGGAGATCA-3' and 5'-AAGCATTTGCGGTGG ACGATGGA-3', respectively. For each assay, a total of $10 \mathrm{uL}$ reaction mixture was prepared using SYBR Green PCR master mix (Applied Biosystems) according to the manufacturer's instructions. Specific cycling conditions for $\beta$-action and F2RL3 were carried out as follow: denaturation at $95^{\circ} \mathrm{C}$ for $3 \mathrm{~min}$, followed by 45 cycles of denaturation at $95^{\circ} \mathrm{C}$ for 20 sec, annealing at $60^{\circ} \mathrm{C}$ for $20 \mathrm{sec}$, extension at $68^{\circ} \mathrm{C}$ for $20 \mathrm{sec}$, and measurement at $80^{\circ} \mathrm{C}$ for $20 \mathrm{sec}$, followed by a final extension at $72^{\circ} \mathrm{C}$ for $5 \mathrm{~min}$. To confirm the specificity of amplification, melting curve analysis were performed and PCR products were sequenced and resolved in a $1 \%$ agarose gel. The F2RL3 mRNA 
expression was represented as $\Delta \mathrm{Ct}=\mathrm{Ct}_{(F 2 R L 3)}-$

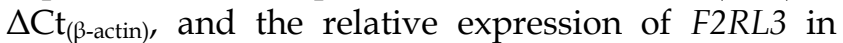
cCRCC was measured using the ratio of F2RL3 expression in ccRCC/matched normal tissues. "Low $F 2 R L 3$ expression" denotes the ratio of F2RL3 mRNA expression in ccRCC/matched normal tissues of less than 3. "Middle F2RL3 expression" denotes the ratio of F2RL3 mRNA expression in ccRCC/matched normal tissues of greater than 3 and less than 6 . "High $F 2 R L 3$ expression" denotes the ratio of F2RL3 mRNA expression in ccRCC/matched normal tissues of greater than 6. Paraffin slices were treated according to the immunohistochemical kit, and results were evaluated by two pathologists independently. Specimens were scored according to the intensity of the dye color. The intensity of the dye color was graded as 0 (no color), 1 (light yellow), 2 (light brown), or 3 (brown). Then specimens were assigned to one of two levels: 0-1 score (-) and 2-3 scores (+).

\section{Statistical analysis}

The associations of F2RL3 expression with clinical-pathological parameters were evaluated using Student's $t$-test for continuous variables and the $\chi^{2}$-test for categorical variables. Progression-free survival (PFS) was measured as the time from the date of surgery to the occurrence of progression, relapse, or death from any cause. Overall survival (OS) was calculated as the time from the initiation of surgery to death from any cause or until the most recent follow-up. Kaplan-Meier method was used for PFS and OS analysis, and different Kaplan-Meier curves were compared by Log-rank test. Using the Cox regression model, univariate and multivariate survival analyses were performed. The level of significance was defined as $p$ value $<0.05$. SPSS software V13.0 (SPSS, Chicago, IL) was used for all statistical analyses.

\section{Results}

The mRNA and protein levels of F2RL3 were examined in 367 couples of ccRCC tissues and cancer-adjacent counterparts. The associations of F2RL3 mRNA and protein levels with clinical features were summarized in Table 1 . We found that F2RL3 mRNA and protein were significant higher in smokers than non-smokers, respectively $(p=0.013$ and 0.033). Importantly, upregulation of F2RL3 mRNA and protein levels significantly correlated with higher $\mathrm{T}$ stage $(p<0.001$ and $<0.001$, respectively), $\mathrm{N}$ stage $(p=0.004$ and 0.002 , respectively), M stage $(p=0.015$ and 0.001 , respectively), and ISUP grade $(p<0.001$ and $<0.001$, respectively). However, no significant differences were observed with regard to the relationship of F2RL3 expression and age at surgery, gender, clinical manifestation, laterality and tumor size $(p>0.05)$. Moreover, the ratio of patients with high F2RL3 expression presented in the group of stage III-IV was higher than in the group of stage I-II $(66.7 \%$ and $57.5 \%$ versus $26.7 \%$ and $35.1 \%$, respectively, all $p<0.001)$, and in group with grade 3-4 was also more than in group with grade $1-2(45.9 \%$ and $51.5 \%$ versus $12.5 \%$ and $23.9 \%$, respectively, all $p<0.001)$ ), as shown in figure 1 . We also found that the mean value of F2RL3 mRNA expression was higher in patients with stage III-IV and grade 3-4, compared with participants with stage I-II and grade $1-2$, respectively (all $p<0.05$, Fig. 2). Meanwhile, the ratio of patients with positive F2RL3 protein expression were found to be higher in stage III-IV and grade 3-4, compared with stage I-II and grade 1-2, respectively (all $\mathrm{p}<0.05$, Fig.3).

Both in univariate and multivariate Cox regression analysis, well-known prognostic factors (e.g. T stage, $\mathrm{N}$ stage, and $\mathrm{M}$ stage) still significantly related to patients' PFS and OS in the cohort of our study, indicating the fine representativeness of population in the current research (Table 2-3). Importantly, high expression of F2RL3 mRNA and protein were observed to be correlated with poor PFS $(p<0.001$ and $=0.003$, respectively) and OS $(p<0.001$ and $=0.009$, respectively) of ccRCC patients in univariate Cox regression analysis (Table 2-3). To eliminate the influence of covariates, F2RL3 expression levels and clinical-pathological factors were further assessed in multivariate Cox regression analysis. Interestingly, high expression of F2RL3 mRNA and protein remained an independently prognostic factor for PFS $(p<0.001$ and $=0.002$, respectively) and OS $(p<0.001$ and $=0.004$, respectively) in multivariate Cox regression model (Table 2-3). With regard to other factors, ISUP grade was detected to be prognostic parameters for PFS in univariate and multivariate Cox regression model, but significant association of ISUP grade with OS was observed in univariate analysis and only a trend was found in multivariate model. Age at surgery was detected to be a prognostic parameter for PFS and OS in univariate but not in multivariate Cox regression model. In addition, gender, smoking, clinical manifestation, laterality and tumor size were not found to be prognostic parameters for patients' PFS and OS in our study (Table 2-3).

As shown in Figure 4, patients with high F2RL3 mRNA levels had a significantly worse PFS and OS $(\mathrm{p}<0.001$ and $<0.001$, respectively), compared with patients with low F2RL3 expression. Non-significant differences between population with low and middle F2RL3 mRNA expression were found for PFS and OS. However, it was interestingly noted that a trend of significant difference in OS between the groups of low 
and middle F2RL3 expression was observed after follow-up of 70 months (Fig. 4). For the association of patients' prognosis with F2RL3 protein expression, we found that the PFS and OS in the patents with positive F2RL3 protein expression were significantly shorten than in negative F2RL3 protein expression, respectively ( $p=0.002$ and $=0.008$, respectively, Fig. 5 ).
In addition, the median PFS in the cohort of patients with low, middle and high F2RL3 expression were 90, 74 and 37 months, respectively. The median OS in patients with low mRNA level of F2RL3 has not yet reached, and in groups with middle and high F2RL3 mRNA expression were 100 and 65 months, respectively (Supplementary Table S1).

Table 1. Clinicopathological characteristics in relation to F2RL3 expression status.

\begin{tabular}{|c|c|c|c|c|c|c|c|c|}
\hline \multirow[t]{2}{*}{ Variable } & \multirow{2}{*}{$\begin{array}{l}\text { Entire group } \\
(n=367)\end{array}$} & \multicolumn{4}{|c|}{ F2RL3 mRNA expression } & \multicolumn{3}{|c|}{ F2RL3 IHC expression } \\
\hline & & $\begin{array}{l}\text { Low expression } \\
(\mathrm{n}=91)\end{array}$ & $\begin{array}{l}\text { Middle expression } \\
(\mathrm{n}=147)\end{array}$ & $\begin{array}{l}\text { High expression } \\
(n=129)\end{array}$ & $\begin{array}{l}P \\
\text { value }^{\mathrm{a}}\end{array}$ & $\begin{array}{l}\text { Negative } \\
(\mathrm{n}=114)\end{array}$ & $\begin{array}{l}\text { Positive } \\
(\mathrm{n}=253)\end{array}$ & $\begin{array}{l}P \\
\text { value }\end{array}$ \\
\hline Age at surgery $(\mathrm{y}$, mean $\pm \mathrm{SD})$ & $55.3 \pm 11.7$ & $55.4 \pm 12.7$ & $55.1 \pm 11.3$ & $55.4 \pm 11.5$ & 0.969 & $55.9 \pm 12.3$ & $55.0 \pm 11.4$ & 0.483 \\
\hline $\operatorname{Sex}(n, \%)$ & & & & & 0.980 & & & 0.464 \\
\hline Male & $248(67.6)$ & $61(67.0)$ & 99 (67.3) & $88(68.2)$ & & $74(64.9)$ & $174(68.8)$ & \\
\hline Female & $119(32.4)$ & $30(33.0)$ & $48(32.7)$ & $41(31.8)$ & & $40(35.1)$ & $79(31.2)$ & \\
\hline Smoking (n, \%) & & & & & 0.013 & & & 0.033 \\
\hline No & $163(44.4)$ & $35(38.5)$ & $79(53.7)$ & $49(38.0)$ & & $60(52.6)$ & $103(40.7)$ & \\
\hline Yes & $204(55.6)$ & $56(61.5)$ & $68(46.3)$ & $80(62.0)$ & & $54(47.4)$ & $150(59.3)$ & \\
\hline Clinical manifestation (n, \%) & & & & & 0.729 & & & 0.562 \\
\hline Incidental & $246(67.0)$ & $60(65.9)$ & $102(69.4)$ & $84(65.1)$ & & $74(64.9)$ & $172(68.0)$ & \\
\hline Symptomatic & $121(33.0)$ & $31(34.1)$ & 45 (30.6) & 45 (34.9) & & $40(35.1)$ & $81(32.0)$ & \\
\hline Laterality $(\mathrm{n}, \%)$ & & & & & 0.902 & & & 0.425 \\
\hline Left & $182(49.6)$ & $44(48.4)$ & $72(49.0)$ & $66(51.2)$ & & $53(46.5)$ & $129(51.0)$ & \\
\hline Right & $185(50.4)$ & 47 (51.6) & $75(51.0)$ & $63(48.8)$ & & $61(53.5)$ & $124(49.0)$ & \\
\hline Tumor size $(\mathrm{cm}$, mean $\pm \mathrm{SD})$ & $5.2 \pm 2.4$ & $4.9 \pm 2.6$ & $5.1 \pm 2.5$ & $5.5 \pm 2.2$ & 0.197 & $4.8 \pm 2.6$ & $5.3 \pm 2.3$ & 0.064 \\
\hline T stage at presentation $(n, \%)$ & & & & & $<0.001$ & & & $<0.001$ \\
\hline $\mathrm{T} 1-\mathrm{T} 2$ & $300(81.7)$ & $84(92.3)$ & $127(86.4)$ & $89(69.0)$ & & $105(92.1)$ & $195(77.1)$ & \\
\hline T3-T4 & $67(18.3)$ & $7(7.7)$ & $20(13.6)$ & $40(31.0)$ & & $9(7.9)$ & $58(22.9)$ & \\
\hline $\mathrm{N}$ stage at presentation $(\mathrm{n}, \%)$ & & & & & 0.004 & & & 0.002 \\
\hline No & $326(88.8)$ & $84(92.3)$ & $137(93.2)$ & $105(81.4)$ & & $110(96.5)$ & $216(85.4)$ & \\
\hline N1 & $41(11.2)$ & $7(7.7)$ & $10(6.8)$ & $24(18.6)$ & & $4(3.5)$ & 37 (14.6) & \\
\hline M stage at presentation $(\mathrm{n}, \%)$ & & & & & 0.015 & & & 0.001 \\
\hline M0 & $330(89.9)$ & $85(93.4)$ & $137(93.2)$ & $108(83.7)$ & & $111(97.4)$ & $219(86.6)$ & \\
\hline M1 & $37(10.1)$ & $6(6.6)$ & $10(6.8)$ & $21(16.3)$ & & $3(2.6)$ & $34(13.4)$ & \\
\hline ISUP grade (n, \%) & & & & & $<0.001$ & & & $<0.001$ \\
\hline $1-2$ & $175(47.7)$ & $63(69.2)$ & $73(49.7)$ & $39(30.2)$ & & 75 (65.8) & $100(39.5)$ & \\
\hline $3-4$ & $192(52.3)$ & $28(30.8)$ & $74(50.3)$ & $90(69.8)$ & & $39(34.2)$ & $153(60.5)$ & \\
\hline
\end{tabular}

aP velue for F2RL3 mRNA expression

$\mathrm{b} P$ velue for F2RL3 IHC expression
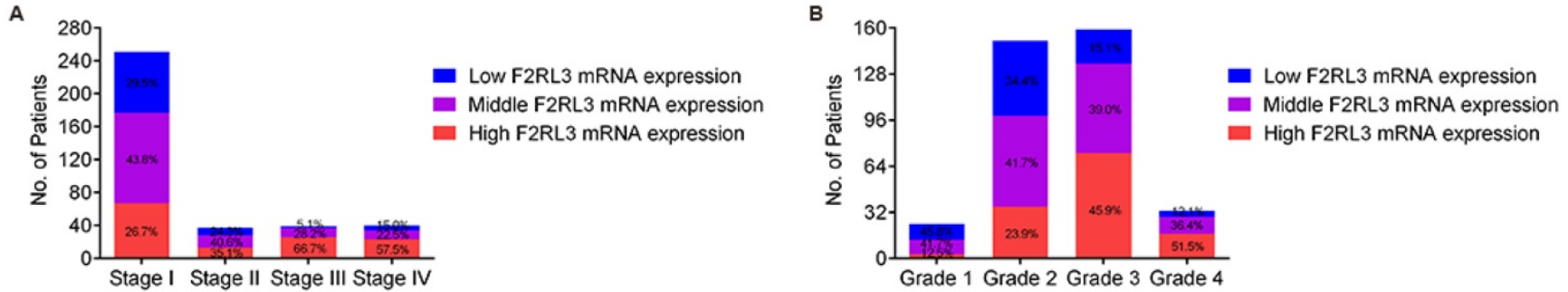

Figure 1. The percentage of high, middle and low F2RL3 mRNA expression in patients with different TNM stage (A) and ISUP grade (B).
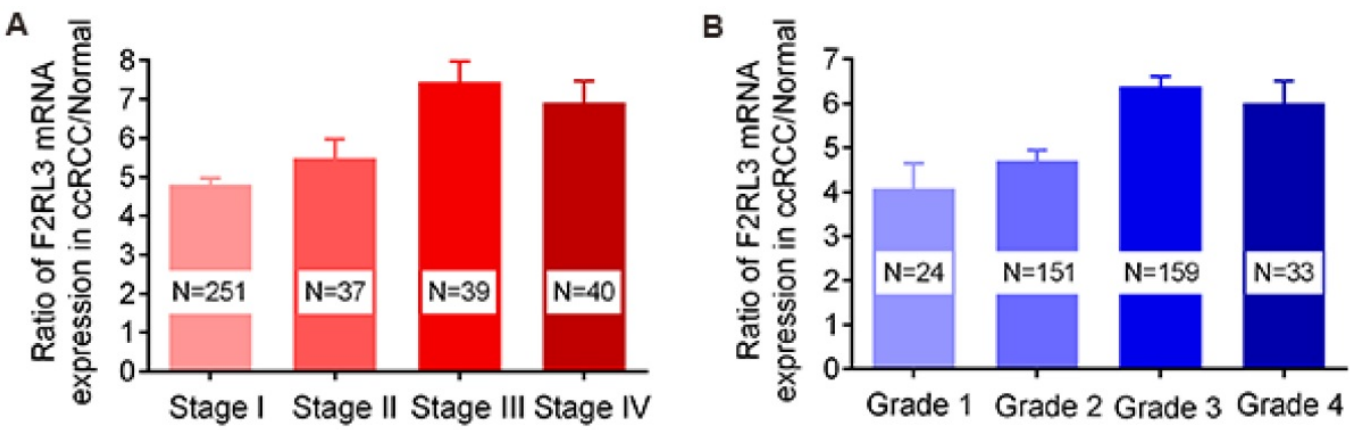

Figure 2. The mean value of F2RL3 mRNA expression in enrolled participants with different TNM stage (A) and ISUP grade (B). 

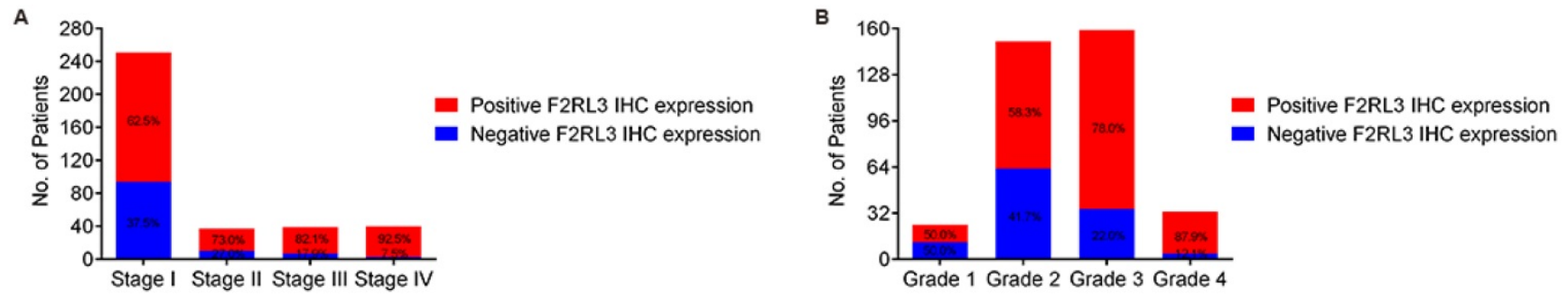

Figure 3. The percentage of positive and negative F2RL3 protein expression in patients with different TNM stage (A) and ISUP grade (B).

Table 2. Univariate and multivariate Cox regression analyses of PFS in 367 enrolled ccRCC patients

\begin{tabular}{|c|c|c|c|c|c|c|}
\hline & Univariate analysis & & Multivariate analysis ${ }^{\mathrm{a}}$ & & Multivariate analysis ${ }^{b}$ & \\
\hline Covariates & HR $(95 \% \mathrm{CI})$ & P value & $\mathrm{HR}(95 \% \mathrm{CI})$ & Pvalue & $\mathrm{HR}(95 \% \mathrm{CI})$ & P value \\
\hline Age at surgery & 1.015(1.003-1.027) & 0.014 & $1.007(0.994-1.020)$ & 0.297 & $1.008(0.995-1.021)$ & 0.240 \\
\hline Sex (male vs. female) & $0.774(0.567-1.058)$ & 0.108 & $0.757(0.514-1.113)$ & 0.157 & $0.765(0.522-1.122)$ & 0.171 \\
\hline Smoking (No vs. Yes) & $1.079(0.813-1.432)$ & 0.599 & $0.856(0.602-1.218)$ & 0.388 & $0.871(0.616-1.230)$ & 0.433 \\
\hline Clinical manifestation (incidental vs. symptomatic) & $1.010(0.751-1.359)$ & 0.945 & $0.965(0.711-1.311)$ & 0.822 & $0.956(0.703-1.299)$ & 0.772 \\
\hline Laterality (left vs. right) & $1.053(0.795-1.393)$ & 0.720 & $1.018(0.765-1.355)$ & 0.902 & $1.031(0.773-1.374)$ & 0.838 \\
\hline Tumor size & $1.033(0.979-1.090)$ & 0.233 & $1.035(0.977-1.096)$ & 0.245 & $1.037(0.980-1.098)$ & 0.211 \\
\hline T stage (T1-T2 vs. T3-T4) & $10.296(7.287-14.549)$ & $<0.001$ & $2.001(1.413-2.832)$ & $<0.001$ & $1.973(1.404-2.772)$ & $<0.001$ \\
\hline N stage (N0 vs. N1) & $12.415(8.356-18.444)$ & $<0.001$ & $2.879(1.677-4.941)$ & $<0.001$ & $2.864(1.672-4.905)$ & $<0.001$ \\
\hline M stage (M0 vs. M1) & $12.324(8.227-18.461)$ & $<0.001$ & $5.047(3.300-7.720)$ & $<0.001$ & $5.116(3.360-7.791)$ & $<0.001$ \\
\hline ISUP grade (1-2 vs. $3-4)$ & $3.019(2.225-4.098)$ & $<0.001$ & $1.866(1.062-3.280)$ & 0.030 & $1.896(1.083-3.319)$ & 0.025 \\
\hline F2RL3 mRNA expression (Low vs. middle) & $1.149(0.777-1.698)$ & 0.487 & $1.159(0.784-1.713)$ & 0.460 & - & - \\
\hline F2RL3 mRNA expression (low vs. high) & $2.060(1.410-3.009)$ & $<0.001$ & $2.113(1.445-3.089)$ & $<0.001$ & - & - \\
\hline F2RL3 IHC expression (negative vs. positive) & $1.657(1.193-2.300)$ & 0.003 & - & - & $1.692(1.218-2.352)$ & 0.002 \\
\hline
\end{tabular}

aMultivariate model include F2RL3 mRNA expression and covariates.

bMultivariate model include F2RL3 IHC expression and covariates.

Table 3. Univariate and multivariate Cox regression analyses of OS in 367 enrolled ccRCC patients

\begin{tabular}{|c|c|c|c|c|c|c|}
\hline & Univariate analysis & & Multivariate analysis ${ }^{a}$ & & Multivariate analysis ${ }^{b}$ & \\
\hline Covariates & $\mathrm{HR}(95 \% \mathrm{CI})$ & Pvalue & $\mathrm{HR}(95 \% \mathrm{CI})$ & Pvalue & $\mathrm{HR}(95 \% \mathrm{CI})$ & P value \\
\hline Age at surgery & $1.018(1.004-1.033)$ & 0.012 & $1.014(0.999-1.029)$ & 0.068 & $1.013(0.998-1.029)$ & 0.086 \\
\hline Sex (male vs. female) & $0.922(0.639-1.331)$ & 0.665 & $1.015(0.635-1.622)$ & 0.952 & $1.028(0.642-1.646)$ & 0.908 \\
\hline Smoking (No vs. Yes) & $1.046(0.745-1.470)$ & 0.794 & $0.968(0.628-1.493)$ & 0.884 & $0.985(0.641-1.516)$ & 0.947 \\
\hline Clinical manifestation (incidental vs. symptomatic) & $0.968(0.674-1.391)$ & 0.861 & $0.951(0.654-1.382)$ & 0.791 & $0.951(0.653-1.384)$ & 0.793 \\
\hline Laterality (left vs. right) & $1.030(0.735-1.443)$ & 0.866 & $0.914(0.645-1.295)$ & 0.613 & $0.938(0.662-1.329)$ & 0.719 \\
\hline Tumor size & $0.995(0.928-1.067)$ & 0.890 & $1.004(0.931-1.082)$ & 0.923 & $1.010(0.938-1.088)$ & 0.792 \\
\hline T stage (T1-T2 vs. T3-T4) & $12.148(8.359-17.654)$ & $<0.001$ & $1.651(1.060-2.573)$ & 0.027 & $1.737(1.120-2.694)$ & 0.014 \\
\hline $\mathrm{N}$ stage (N0 vs. N1) & $12.684(8.352-19.264)$ & $<0.001$ & $3.147(1.777-5.571)$ & $<0.001$ & $2.972(1.674-5.276)$ & $<0.001$ \\
\hline M stage (M0 vs. M1) & 11.554 (7.618-17.525) & $<0.001$ & $5.456(3.377-8.813)$ & $<0.001$ & $5.858(3.640-9.428)$ & $<0.001$ \\
\hline ISUP grade (1-2 vs. $3-4)$ & $3.466(2.364-5.038)$ & $<0.001$ & $1.658(0.938-2.930)$ & 0.082 & $1.612(0.909-2.858)$ & 0.102 \\
\hline F2RL3 mRNA expression (Low vs. middle) & $1.473(0.875-2.479)$ & 0.145 & $1.497(0.889-2.520)$ & 0.129 & - & - \\
\hline F2RL3 mRNA expression (low vs. high) & $2.826(1.713-4.662)$ & $<0.001$ & $2.936(1.777-4.851)$ & $<0.001$ & - & - \\
\hline F2RL3 IHC expression (negative vs. positive) & $1.712(1.140-2.569)$ & 0.009 & - & - & $1.811(1.203-2.725)$ & 0.004 \\
\hline
\end{tabular}

aMultivariate model include F2RL3 mRNA expression and covariates.

bMultivariate model include F2RL3 IHC expression and covariates.


Figure 4. Correlated of F2RL3 mRNA expression with PFS (A) and OS (B) in the enrolled 367 ccRCC patients. High F2RL3 mRNA expression is significantly associated with poor PFS and OS, compared with middle and low F2RL3 expression. 

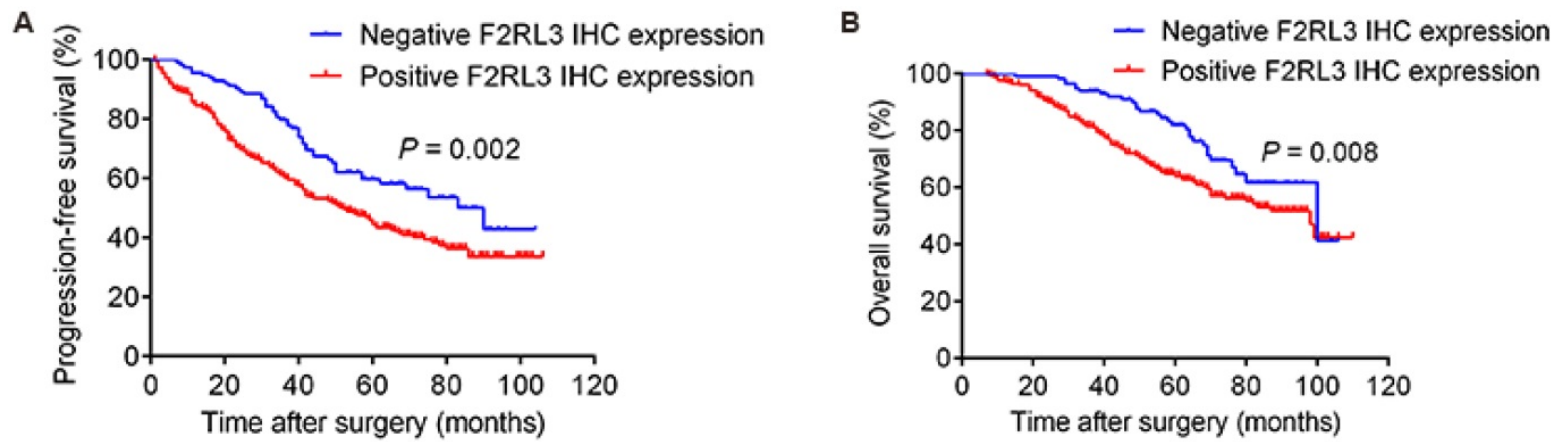

Figure 5. Correlated of F2RL3 protein expression with PFS (A) and OS (B) in the enrolled 367 ccRCC patients. The PFS and OS in the patents with positive F2RL3 protein expression were significantly shorten than in negative F2RL3 protein expression

\section{Discussion}

The current study indicated that upregulated expression of F2RL3 contributes to progressive characteristics and poor prognosis of ccRCC. More importantly, the association of elevated expression of F2RL3 with PFS and OS of ccRCC patients persisted after adjusting for a variety of confounding factors (e.g. smoking-exposure).

For kidney cancer, about $20-30 \%$ patients are presented with metastatic disease at the time of diagnosis ${ }^{[17]}$. Meanwhile, another $20 \%$ of all patients received nephrectomy will have a relapse and develop metastatic RCC during follow-up ${ }^{[18]}$. The fact that the prognosis of metastatic RCC is poor ${ }^{[17]}$ makes the occurrence of metastatic RCC a serious burden for oncologic healthcare worldwide. Specific lifestyle factors including tobacco-exposure are vital etiologic factors for RCC[3]. Investigating the association of tobacco-exposure related gene with RCC incidence and prognosis extend our comprehension of mechanisms by which smoking-related gene increase risk of RCC and we have potential to explore novel therapeutic interventions.

The F2RL3 gene, which codes for the thrombin PAR-4, was strong associated with smoking in previous studies[12-14]. It should be importantly noted that PAR4 has been suggested to be involved in the development of several carcinoma ${ }^{[19,}$ 20] and metastasis ${ }^{[21]}$. For example, Baglietto et al ${ }^{[20]}$ found that smoking related F2RL3 methylation changes measurable in peripheral blood may improve prediction of lung cancer risk. In addition, a study conducted by Zhang et al[16] indicated that F2RL3 methylation is a strong predictor of cancer mortality and pathways associated with F2RL3 methylation may regulate harmful effects of smoking. Moreover, PAR-4 is a thrombin receptor and regulate blood coagulation processes ${ }^{[22]}$. Hyper-coagulation is a common feature in patients with cancer, which from other side provides plausible explanation for the relationship of $F 2 R L 3$ gene and carcinogenesis.
The main question proposed in this study was whether mRNA changes of smoking-related F2RL3 gene are involved in ccRCC prognosis. Although our data is impossible to deeply answer the question such as its underlying mechanism, the results of our study indicate the close association of increased F2RL3 mRNA level with progressive feature and poor prognosis of ccRCC. In the current study, we observed that high mRNA expression of F2RL3 present in patients with T3-4, N1, M1, or ISUP grade 3-4. Meanwhile, well-known prognostic factors such as TNM stage and ISUP grade still significantly correlated with PFS and OS in the cohort of this study, which are consistent with a variety of guidelines and previous studies ${ }^{[23]}$. This supports that high F2RL3 expression associated with advanced TNM stage or ISUP grade is an adverse prognostic factor for ccRCC. Furthermore, we confirmed that elevated F2RL3 mRNA expression are associated with ccRCC poor survival after adjustment for smoking status and so on. It may partly reflect that smoking-exposure can result in various changes in cells, of which only some vital variations will trigger carcinogenesis. We speculated that smoking-exposure leads to higher expression of F2RL3 in cells and clonal reproduction, this anomaly in upregulated F2RL3 expression persists and maintains the stability of mitosis through subsequent cell divisions. Analyzing the association of F2RL3 gene methylation status and protein expression with patients' prognosis and building a prediction model based on F2RL3 mRNA/protein measured in blood samples are required for fully understand the value of this gene in ccRCC. Additional investigations such as a mechanism analysis are necessary for the full assessment of the causal relevance of F2RL3 in ccRCC development (work in preparation).

In conclusion, our study indicates that high expression of smoking-related F2RL3 gene is correlated with progressive characteristics and worse prognosis of ccRCC. It provides a basic message for the notion that elevated expression of F2RL3 in ccRCC 
may be crucial to gain the aggressive features and poorly prognostic phenotype. These findings encourage further work to explore the potential function of F2RL3 in ccRCC development.

\section{Supplementary Material}

Supplementary table.

http://www.jcancer.org/v09p3400s1.pdf

\section{Acknowledgements}

We would like to thank all laboratory members for their critical discussion of this manuscript, and apologize to those not mentioned due to space limitations.

\section{Funding}

This work was supported by grants from the 863 Program (number 2015AA020913), the 973 Program (Number 2015CB943300), the National Science Foundation of China (Numbers 81471454 and 31521003), Commission for Science and Technology of Shanghai Municipality (Numbers 14ZR1402000 and 15XD1500500) to Jianyuan Zhao; grant from the National Science Foundation of China (Number 81302213) to Dalong Cao; grants from Shanghai Sailing Program (Number 17YF1402700) and State Key Laboratory of Genetic Engineering (Number SKLGE-1605) to Yuanyuan Qu; grant from Shanghai rising star project (Number 16QA1401100) to Yao $\mathrm{Zhu}$; and grant from the National Science Foundation of China (Number 81502195) to Guiming Zhang.

\section{Authors' contributions}

DLC, YYQ, and XZ performed the experiments, wrote and revised the paper, collected clinical data and analyzed the data. FJX, SXZ, GMZ, BD, YZ, GHS, YJS and YPZ revised the paper and collected the clinical data. HLZ, DWY and JYZ guided the experimental design and revised the manuscript. All authors read and approved the final the manuscript.

\section{Competing Interests}

The authors have declared that no competing interest exists.

\section{References}

[1] Ferlay J, Soerjomataram I, Dikshit R, et al. Cancer incidence and mortality worldwide: Sources, methods and major patterns in GLOBOCAN 2012[J]. INT J CANCER. 2015,136(5): E359-E386.

[2] Mathew A, Devesa SS, Fraumeni JJ, et al. Global increases in kidney cancer incidence, 1973-1992[J]. EUR J CANCER PREV. 2002,11(2): 171-178.

[3] Hunt JD, van der Hel OL, McMillan GP, et al. Renal cell carcinoma in relation to cigarette smoking: Meta-analysis of 24 studies[J]. INT J CANCER. 2005,114(1): 101-108.

[4] Theis RP, Dolwick GS, Burr D, et al. Smoking, environmental tobacco smoke, and risk of renal cell cancer: A population-based case-control study[J]. BMC CANCER. 2008,: 387.

[5] $\mathrm{Hu}$ J, Ugnat AM. Active and passive smoking and risk of renal cell carcinoma in Canada[J]. EUR J CANCER. 2005,41(5): 770-778.
[6] Sweeney C, Farrow DC. Differential survival related to smoking among patients with renal cell carcinoma[J]. EPIDEMIOLOGY. 2000,11(3): 344-346.

[7] Kroeger N, Klatte T, Birkhauser FD, et al. Smoking negatively impacts renal cell carcinoma overall and cancer-specific survival[J]. CANCER-AM CANCER SOC. 2012,118(7): 1795-1802.

[8] US CFDC, US NCFC, US OOSA. How tobacco smoke causes disease: The biology and behavioral basis for Smoking-Attributable disease: A report of the surgeon general[M]. Atlanta (GA): Centers for Disease Control and Prevention (US), 2010.

[9] Clague J, Shao L, Lin J, et al. Sensitivity to NNKOAc is associated with renal cancer risk[J]. CARCINOGENESIS. 2009,30(4): 706-710.

[10] Leger AJ, Covic L, Kuliopulos A. Protease-activated receptors in cardiovascular diseases[J]. CIRCULATION. 2006,114(10): 1070-1077.

[11] Wu CC, Teng CM. Comparison of the effects of PAR1 antagonists, PAR4 antagonists, and their combinations on thrombin-induced human platelet activation[J]. EUR J PHARMACOL. 2006,546(1-3): 142-147.

[12] Shenker NS, Polidoro S, van Veldhoven K, et al. Epigenome-wide association study in the European Prospective Investigation into Cancer and Nutrition (EPIC-Turin) identifies novel genetic loci associated with smoking[J]. HUM MOL GENET. 2013,22(5): 843-851.

[13] Sun YV, Smith AK, Conneely KN, et al. Epigenomic association analysis identifies smoking-related DNA methylation sites in African Americans[J]. HUM GENET. 2013,132(9): 1027-1037.

[14] Elliott HR, Tillin T, McArdle WL, et al. Differences in smoking associated DNA methylation patterns in South Asians and Europeans[J]. CLIN EPIGENETICS. 2014,6(1): 4.

[15] Zhang Y, Yang R, Burwinkel B, et al. F2RL3 methylation as a biomarker of current and lifetime smoking exposures[J]. Environ Health Perspect. 2014,122(2): 131-137.

[16] Zhang Y, Yang R, Burwinkel B, et al. F2RL3 methylation in blood DNA is a strong predictor of mortality[J]. INT J EPIDEMIOL. 2014,43(4): 1215-1225.

[17] Gupta K, Miller JD, Li JZ, et al. Epidemiologic and socioeconomic burden of metastatic renal cell carcinoma (mRCC): A literature review[J]. CANCER TREAT REV. 2008,34(3): 193-205.

[18] Athar U, Gentile TC. Treatment options for metastatic renal cell carcinoma: A review[J]. CAN J UROL. 2008,15(2): 3954-3966.

[19] Black PC, Mize GJ, Karlin P, et al. Overexpression of protease-activated receptors-1,-2, and-4 (PAR-1, -2 , and -4 ) in prostate cancer[J]. PROSTATE. 2007,67(7): 743-756.

[20] Baglietto L, Ponzi E, Haycock P, et al. DNA methylation changes measured in pre-diagnostic peripheral blood samples are associated with smoking and lung cancer risk[J]. INT J CANCER. 2017,140(1): 50-61.

[21] Kaufmann R, Rahn S, Pollrich K, et al. Thrombin-mediated hepatocellular carcinoma cell migration: Cooperative action via proteinase-activated receptors 1 and 4[J]. J CELL PHYSIOL. 2007,211(3): 699-707.

[22] Elste AP, Petersen I. Expression of proteinase-activated receptor 1-4 (PAR 1-4) in human cancer[J]. J MOL HISTOL. 2010,41(2-3): 89-99.

[23] Keegan KA, Schupp CW, Chamie K, et al. Histopathology of surgically treated renal cell carcinoma: Survival differences by subtype and stage[J]. J Urol. 2012,188(2): 391-397. 